\title{
Decreased S10oB expression in chronic liver diseases
}

\author{
Su Jung Baik ${ }^{1}$ Tae Hun Kim², Kwon $\mathrm{Yoo}^{2}$, Il Hwan Moon ${ }^{2}$, Ju Young Choi ${ }^{2}$, Kyu Won Chung ${ }^{3}$, and \\ Dong Eun Song 4
}

\begin{abstract}
${ }^{1}$ Healthcare Research Team, Health Promotion Center, Gangnam Severance Hospital, Yonsei University College of Medicine, Seoul; ${ }^{2}$ Department of Internal Medicine, Ewha Womans University School of Medicine, Seoul; ${ }^{3}$ Department of Hepatobiliary, College of Medicine, Incheon St. Mary's Hospital, The Catholic University of Korea, Incheon; ${ }^{4}$ Department of Pathology, Asan Medical Center, University of Ulsan College of Medicine, Seoul, Korea
\end{abstract}

Received: July 10, 2015

Revised : August 24, 2015

Accepted: August 29, 2015

\section{Correspondence to}

Tae Hun Kim, M.D.

Division of Gastroenterology and Hepatology, Department of Internal Medicine, Ewha Womans University School of Medicine, 1071 Anyangcheon-ro,

Yangcheon-gu, Seoul 07985, Korea

Tel: +82-2-2019-1290

Fax: +82-2-2019-4802

E-mail: thkm@ewha.ac.kr
Background/Aims: Hepatic innervation in liver diseases is not fully understood. We here evaluated S10oB expression as a marker of hepatic nerves in patients with various chronic liver diseases, topographically and semi-quantitatively.

Methods: Liver specimens were obtained from 70 subjects (three controls, and 32 chronic hepatitis B, 14 chronic hepatitis C, 14 liver cirrhosis, and seven hepatocellular carcinoma patients). The hepatic nerve density was calculated based on immunohistochemical staining of $\mathrm{S} 100 \mathrm{~B}$ protein in the portal tracts and hepatic lobules. S10oB mRNA levels were semi-quantitatively assessed as the S10oB/glyceraldehyde 3-phosphate dehydrogenase (GAPDH) mRNA ratio.

Results: The densities of the hepatic nerves in portal tracts of chronic liver diseases were not significantly different from those of normal controls but the hepatic nerve densities in lobular areas of liver cirrhosis were significantly decreased $(p=0.025)$. Compared to the control, the S100B/GAPDH mRNA ratio was significantly decreased in chronic liver diseases $(p=0.006)$ and most decreased in chronic hepatitis $\mathrm{C}$ patients $(p=0.023)$. In chronic liver diseases, The $S_{100 B /}$ GAPDH mRNA ratio tended to decrease as the fibrosis score $>0(p=0.453)$ but the

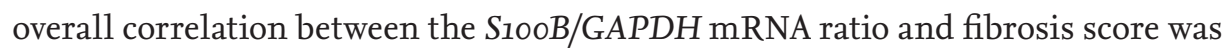
not statistically significant $(r=0.061, p=0.657)$.

Conclusions: Hepatic innervation is decreased in cirrhotic regenerating nodules compared to the control group and seems to decrease in early stages of fibrosis progression. Further studies are needed to clarify the association between changes of hepatic innervation and chronic liver disease progression.

Keywords: Chronic liver disease; Fibrosis; Hepatitis; Nerve fibers; S1oo proteins

\section{INTRODUCTION}

The human liver receives sympathetic input via the celiac plexus and parasympathetic input mainly from the vagus nerve [1]. Hepatic nerve fibers are found around the hepatic arteriole, portal vein, bile duct in the portal tract, and central veins inside the hepatic lobule along the sinusoid [2]. The hepatic nerve is involved in hemodynamics, bile flow regulation, as well as in carbohydrate and lipid metabolism $[3,4]$. Activation of the sympathetic nerves increases glucose and lactate release, bile flow, and bile acid secretion [5], and sympathetic nervous overactivity is related to the severity of cirrhosis [3].

The S10o protein is a low molecular weight (9 to $13 \mathrm{kDa}$ ) calcium $\left(\mathrm{Ca}^{2+}\right)$-binding protein of the helix-loop-helix type [6], which acts as a serum marker for brain damage in cirrhosis with hepatic encephalopathy $[7,8]$. The nervous system-specific S10oB isoform is a cytoplasmic 
component of glial cells [9,10], and marks hepatic nerve fibers in the portal area [11].

In chronic liver disease, the distribution of intrahepatic nerve fibers is altered, accompanying structural changes in the hepatic lobules [2]; however, changes in hepatic innervation in chronic liver diseases has not yet been fully elucidated. We here aimed to evaluate the density of hepatic nerves in various chronic liver diseases, topographically and semi-quantitatively.

\section{METHODS}

\section{Tissue specimens}

The study was approved by the Institutional Review Boards of Ewha Womans University Mokdong Hospital (EUMC 2015-03-026-001). All patients gave informed consent. We included samples from three normal controls and 67 patients with chronic liver diseases 32 chronic hepatitis B [CHB], 14 chronic hepatitis C [CHC], 14 liver cirrhosis [LC], and seven hepatocellular carcinoma [HCC] patients). Normal liver tissues were obtained from two patients undergoing laparoscopic cholecystectomy, one patient undergoing angiosarcoma lobectomy. Liver tissues were obtained by ultrasonography-guided needle biopsy or surgical wedge resection at Ewha Womans University Mokdong Hospital from May 2006 to July 2008. Biopsy was done before anti-viral therapy started in patient with viral hepatitis. Chronic hepatitis was considered as CHB and CHC. The following were considered as chronic liver diseases: $\mathrm{CHB}, \mathrm{CHC}$, and LC. Fibrosis score was analyzed from 56 patients $(28 \mathrm{CHB}, 14$ $\mathrm{CHC}$, and 14 LC). The necroinflammation score and fibrosis score were determined in terms of Knodell's histological activity index (HAI) [12].

\section{S100B immunohistochemistry}

Immunohistochemistry was performed using the LSAB System/HRP kit (DaKoCytomation, Carpinteria, CA, USA). Endogenous peroxidase activity was blocked by incubation in ready-to-use peroxidase blocking agent (DaKoCytomation). Non-specific protein binding was blocked by incubating tissues with Protein Block Serum-free (DaKoCytomation). Tissues were incubated with the polyclonal rabbit anti-S10oB antibody (1:80o dilution; DaKoCytomation, Copenhagen, Denmark). Subsequently, the tis- sues were incubated for 15 minutes with biotinylated anti-rabbit immunoglobulins in phosphate-buffered saline, followed by incubation with streptavidin peroxidase for 15 minutes. 3, 3'-Diaminobenzidine tetrachloride (Liquid DAB Substrate Chromogen System, DaKoCytomation) was used as substrate for the color reaction and was counterstained with Mayer's hematoxylin. The stained tissues were then photographed using an Olympus microscope equipped with a ColorView3 digital camera (Soft Imaging System GmbH, Münster, Germany).

\section{Hepatic nerve density}

S10oB-positive spindle-like nerve fibers were counted at the portal tracts and the hepatic lobules. The S1ooB-positive nerve fibers were counted at least 2 high-power fields per specimen. One to five specimens per patient were evaluated. The mean number of stained nerve fibers per portal tract and hepatic lobule was calculated. In patients with LC, hepatic nerves were calculated at fibrous septae and regenerating nodules. The hepatic nerve density in the hepatic lobule was compared to that in the regenerating nodules in LC patients. In HCC patients, some S1ooB-stained fibers considered to be hepatic nerve fibers were found in stroma and tumorous tissue. Because tumor stroma and tumor parenchyma could not be clearly distinguished in some specimens, we present hepatic nerve density of HCC in the table but

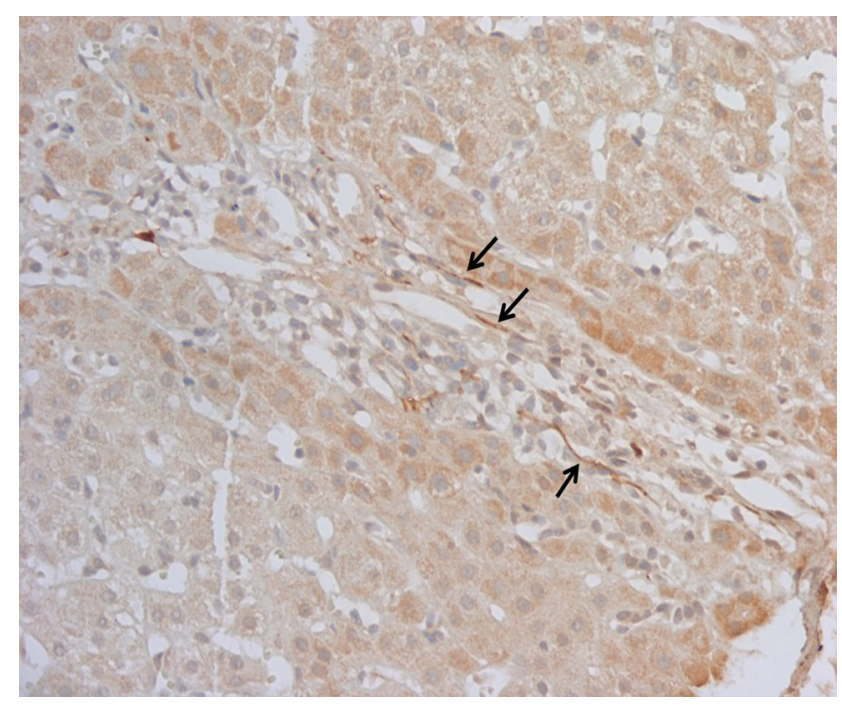

Figure 1. Distribution of nerve fibers in immunohistochemical stain. S100-positive nerve fibers are seen in the portal tract (arrows; liver cirrhosis patient, $\times 100$ ). 
Table 1. Primers for S10oB and GAPDH

\begin{tabular}{|c|c|c|c|}
\hline Transcript & Primer pair & Sequence & Base pair \\
\hline \multirow[t]{2}{*}{ S100B } & Forward & 5'-ATG TCT GAG CTG GAG AAG G-3' & 338 \\
\hline & Reverse & 5'-CTG TCT GCT TTC TTG CAT G-3' & \\
\hline \multirow[t]{2}{*}{ GAPDH } & Forward & 5'-TGA TGA CAT CAA GAA GGT GGT GAA G-3' & 240 \\
\hline & Reverse & 5'-TCC TTG GAG GCC ATG TGG GCC AT-3’ & \\
\hline
\end{tabular}

GAPDH, glyceraldehyde 3-phosphate dehydrogenase.

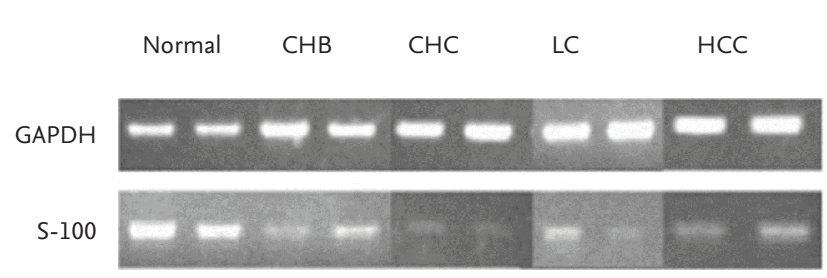

Figure 2. Polymerase chain reaction (PCR) expression of $S_{100 B}$ mRNA. PCR expression of $S_{100 B}$ mRNA was decreased in patients with chronic liver diseases. CHB, chronic hepatitis B; CHC, chronic hepatitis C; LC, liver cirrhosis; HCC, hepatocellular carcinoma; GAPDH, glyceraldehyde 3-phosphate dehydrogenase.

excluded the density from statistical analysis.

\section{S10oB mRNA reverse transcription polymerase chain reaction}

For mRNA preparation, the tissues were incubated in easy-BLUE (80o mL; European Biotech Network, Dolembreux, Belgium) for 5 minutes at room temperature. After adding $200 \mu \mathrm{L}$ chloroform, the tissues were vortexed. The tissues were centrifuged at 12,000 rpm for $15 \mathrm{~min}-$ utes at $4^{\circ} \mathrm{C}$. To the supernatant, $400 \mathrm{~mL}$ isopropyl alcohol was added and mixed by inverting two or three times. The mixture was incubated for 5 minutes at room temperature, and centrifuged at $12,000 \mathrm{rpm}$ for $10 \mathrm{~min}-$ utes at $4^{\circ} \mathrm{C}$. After centrifugation, the precipitated RNA was washed with ethanol, dried, and resuspended in 25 $\mathrm{mL}$ of diethylpyrocarbonate-treated distilled water before being stored at $-70^{\circ} \mathrm{C}$ until required; the RNA was spectrophotometrically quantitated (Ultrospec 210opro, Amersham Biosciences, Little Chalfont, England).

RNA was reverse transcribed into cDNA using an oligo-dT primer and M-MLV reverse transcriptase (Promega, Madison, WI, USA), in the presence of rRNasin Ribonuclease Inhibitor (Promega). Glyceraldehyde 3-phosphate dehydrogenase (GAPDH; as house keeping gene) and $S_{100 B}$ mRNAs were amplified using gene-specific primer sets (COSMO Genetech, Seoul, Korea) (Table 1) and Taq polymerase (Promega) on a GeneAmp PCR System 9600 (Perkin-Elmer Corp., Norwalk, CT, USA). The products were subjected to $2 \%$ agarose gel electrophoresis (Mupid a, Advance, Kasugai, Japan) and band density (Fig. 2) measured using a GelDoc 2000 system (Bio-Rad, Hercules, CA, USA) and Quantity One software (Bio-Rad).

\section{Statistical analysis}

The differences in the means between groups were determined using analysis of variance and Student $t$ test. The differences of median between groups were determined by non-parametric test (Kruskal-Wallis test or Wilcoxon rank-sum test). In non-parametric tests, significance of differences between groups was analyzed by the Dunn procedure. Correlation between semi-quantitative $S_{100 B}$ mRNA levels and the degree of fibrosis was calculated using Spearman correlation coefficient. $p$ values $<0.05$ were considered as statistically significant. By the Dunn procedure, $p$ values $<0.01$ were considered as statistically significant. Analyses were performed using the R Statistical Package version 3.1.2, (Institute for Statistics and Mathematics, Vienna, Austria, www.r-project. org) and SAS version 9.2 (SAS Institute Inc., Cary, NC, USA).

\section{RESULTS}

\section{Patient characteristics}

Patient characteristics are summarized in Table 2. Mean age was younger in CHB (37.5 years) and older in HCC patients (62.4 years, $p<0.0001)$. Level of alanine aminotransferase and aspartate aminotransferase between groups was not significantly different. The HAI score 
Table 2. Characteristics of patients

\begin{tabular}{|c|c|c|c|c|c|c|}
\hline Characteristic & $\begin{array}{c}\text { Controls } \\
(\mathrm{n}=3)\end{array}$ & $\begin{array}{c}\text { CHB } \\
(n=32)\end{array}$ & $\begin{array}{c}\text { CHC } \\
(n=14)\end{array}$ & $\begin{array}{c}\text { LC } \\
(n=14)\end{array}$ & $\begin{array}{l}\text { HCC } \\
(n=7)\end{array}$ & $p$ value \\
\hline Age, yr & $43.0 \pm 7.0$ & $37.5 \pm 13.8$ & $49.9 \pm 14.0$ & $49.7 \pm 8.7$ & $62.4 \pm 12.4$ & $<0.0001$ \\
\hline AST, IU/L & - & 164.2 & 85.8 & 167.2 & 58.3 & \\
\hline ALT, IU/L & - & $253 \cdot 3$ & 133.1 & 114.8 & 30.3 & \\
\hline Total bilirubin, mg/dL & - & 1.0 & 0.9 & 1.9 & 1.1 & \\
\hline Direct bilirubin, mg/dL & - & 0.5 & 0.4 & 0.9 & 0.5 & \\
\hline HAI score & - & $8.21 \pm 4.03$ & $6.86 \pm 2.32$ & $11.29 \pm 3.54$ & - & $0.005^{\mathrm{a}}$ \\
\hline Fibrosis score & - & $1.75 \pm 1.14$ & $1.21 \pm 0.80$ & $3.50 \pm 0.85$ & - & $<0.0001^{b}$ \\
\hline Median Fibrosis score & - & 1 & 1 & 4 & - & $<0.0001^{\mathrm{c}}$ \\
\hline
\end{tabular}

Values are presented as mean \pm SD. The fibrosis score were determined in terms of Knodell's HAI.

CHB, chronic hepatitis B; CHC, chronic hepatitis C; LC, liver cirrhosis; HCC, hepatocellular carcinoma; AST, aspartate aminotransferase; ALT, alanine aminotransferase; HAI, histological activity index.

${ }^{\mathrm{a}} \mathrm{CHB}$ vs. LC, $p=0.029$; CHC vs. LC, $p=0.005$.

${ }^{\mathrm{b}} \mathrm{CHB}$ vs. LC, $p<0.0001 ; \mathrm{CHC}$ vs. LC, $p<0.0001$.

${ }^{\mathrm{c}} \mathrm{CHB}$ vs. LC, $p=0.0004 ; \mathrm{CHC}$ vs. LC, $p<0.0001$.

Table 3. Semi-quantitation of $S 100 B$ mRNA and hepatic nerve density in the portal tract and hepatic lobule

\begin{tabular}{|c|c|c|c|c|c|c|}
\hline Variable & Control & $\mathrm{CHB}$ & $\mathrm{CHC}$ & $\mathrm{LC}$ & HCC & $p$ value \\
\hline Semi-quantitation of $S_{100 B}$ mRNA & 3 & 32 & 14 & 14 & 7 & \\
\hline$S_{100 B}$ & $1,689.9$ & $1,427.6$ & $1,463.0$ & $1,623.1$ & $1,438.4$ & \\
\hline GAPDH & $1,413 \cdot 4$ & $1,534.6$ & $1,681.5$ & $1,766.7$ & $1,672.3$ & \\
\hline \multicolumn{7}{|l|}{ S10oB/GAPDH ratio } \\
\hline Mean \pm SD & $1.21 \pm 0.07$ & $0.94 \pm 0.12$ & $0.88 \pm 0.12$ & $0.93 \pm 0.12$ & $0.86 \pm 0.09$ & 0.0007 \\
\hline Mean \pm SD & \multicolumn{3}{|c|}{$0.92 \pm 0.12^{\mathrm{a}}$} & & & 0.0002 \\
\hline Median & 1.23 & 0.93 & $0.88^{\mathrm{b}}$ & 0.92 & $0.89^{b}$ & 0.023 \\
\hline Median & \multicolumn{3}{|c|}{$0.91^{\mathrm{a}}, 0.91^{\mathrm{c}}$} & & & $<0.05^{\mathrm{d}}$ \\
\hline Hepatic nerve density by S1ooB stain & 2 & 14 & 12 & 10 & 7 & \\
\hline Median portal tract & $3 \cdot 42$ & 2.50 & 1.00 & 1.75 & 2.0 & $0.158^{e}$ \\
\hline Median hepatic lobule & 1.42 & 0.50 & 0.33 & $0.25^{f}$ & 1.5 & $0.231^{\mathrm{e}}$ \\
\hline
\end{tabular}

CHB, chronic hepatitis B; CHC, chronic hepatitis C; LC, liver cirrhosis; HCC, hepatocellular carcinoma; GAPDH, glyceraldehyde 3-phosphate dehydrogenase.

${ }^{\mathrm{a}} \mathrm{CH}$, chronic hepatitis (CHB, CHC).

${ }^{\mathrm{b}}$ Control vs. CHC, $p=0.002$; Control vs. HCC, $p=0.003$.

${ }^{\mathrm{c}} \mathrm{CLD}$, chronic liver diseases (CHB, CHC, and LC).

${ }^{\mathrm{d}}$ Control vs. $\mathrm{CH}, \mathrm{p}=0.0008$; Control vs. CLD, $\mathrm{p}=0.006$.

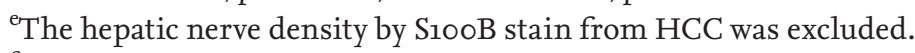

${ }^{\mathrm{f}}$ Control vs. LC, $p=0.025$.

was significantly highest in LC patients $(p=0.005)$. Fibrosis scores (CHB vs. CHC vs. LC; $1.7 \pm 1.1,1.2 \pm 0.8,3.5 \pm$ $0.9, p<0.0001$ ) were significantly highest in LC patients (Table 2).The difference in the fibrosis score was not statistically significant between $\mathrm{CHB}$ and $\mathrm{CHC}$ patients ( $p$ $=0.24$ ).

\section{Hepatic nerve density}

In normal controls, S10oB-positive nerve fibers were located in the portal tracts, and along the sinusoids in the hepatic lobules. In CHB and CHC patients, they were found in the portal tracts, but were rare in hepatic lobules. In LC patients, nerve fibers were noted in fibrous septae, but were rare in regenerating nodules (Fig. 1). 


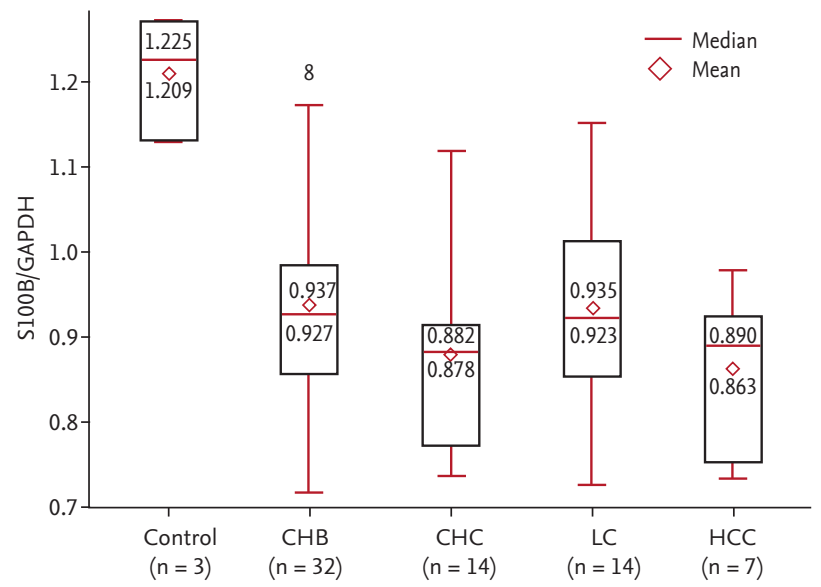

Figure 3. S100B/glyceraldehyde 3-phosphate dehydrogenase $(G A P D H)$ mRNA ratio in control and various liver diseases.

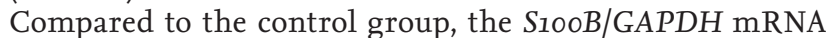
ratio was significantly decreased in chronic liver diseases

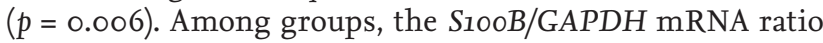
was significantly decreased in chronic hepatitis $\mathrm{C}$ (CHC) patients $(p=0.023)$. CHB, chronic hepatitis B; LC, liver cirrhosis; HCC, hepatocellular carcinoma.

The hepatic nerve density in the portal tracts and hepatic lobules was decreased in patients with chronic liver diseases overall. In the hepatic lobular area, the nerve densities of cirrhotic regenerating nodules were significantly lower than the lobular nerve densities of control patients $(p=0.025)$ (Table 3$)$. When comparing the hepatic nerve densities of each chronic liver diseases, there were no differences in either portal tracts $(p=0.158)$ or hepatic lobules $(p=0.231)$.

\section{Semi-quantitation of S100B mRNA (S100B/GAPDH mRNA ratio)}

The S100B/GAPDH mRNA ratio ranged from 0.717 to 1.27. The $S 100 B / G A P D H$ mRNA ratio was decreased in patients with chronic liver diseases $(p=0.006$ ) (Fig. 3). This ratio was significantly decreased in CHC among groups $(p=0.023)$ (Table 3 ). The S10oB/GAPDH mRNA ratio was significantly decreased in $\mathrm{CHC}$ (vs. control, $p$ $=0.002$ ) and in HCC patients (vs. control, $p=0.003$ ). The S10oB/GAPDH mRNA ratio of chronic hepatitis patients overall was lower than that of the control group (1.22 vs. $0.91, p=0.006$ ), although CHC patients had a non-significantly lower $S 100 B / G A P D H$ mRNA ratio than CHB patients $(p=0.12)$. In chronic liver disease patients, the S10oB/GAPDH mRNA ratio tended to decrease as the

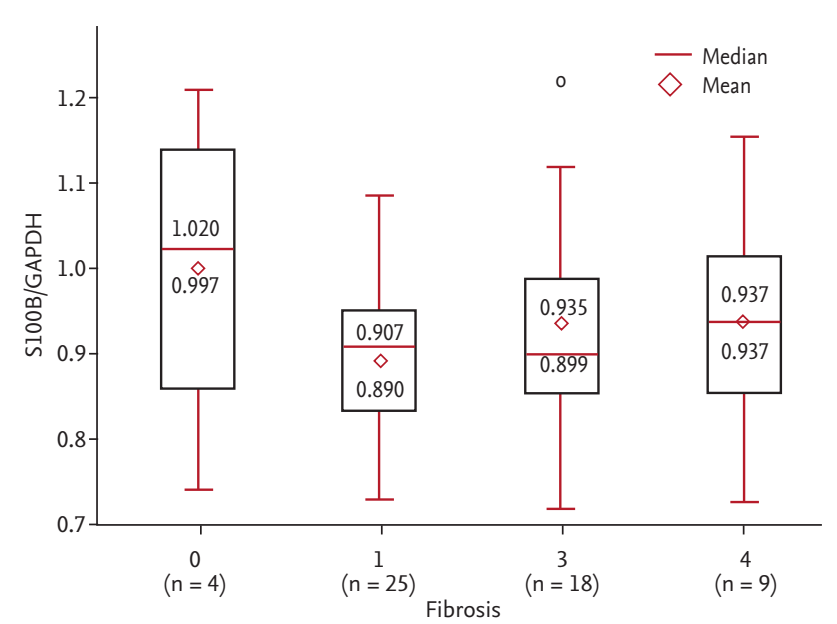

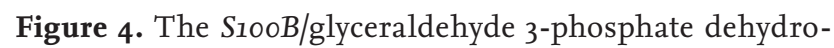
genase (GAPDH) mRNA ratio and fibrosis scores in chronic liver diseases. In chronic liver diseases, The $S_{100 B / G A P D H}$ mRNA ratio tended to decrease as the fibrosis score $>0(p=$ 0.453), but the overall correlation between the S10oB/GAPDH mRNA ratio and fibrosis score was not statistically significant $(r=0.061, p=0.657) \cdot r(r h o)$, Spearman correlation coefficient.

fibrosis score increased, but the trend was not statistically significant $(p=0.453$ ) (Fig. 4$)$. There was no statistically significant correlation between the S100B/GAPDH mRNA ratio and the fibrosis score $(r=0.061, p=0.657)$.

\section{DISCUSSION}

The human liver is innervated by the sympathetic and parasympathetic nervous systems [1]. The nerve fibers end on hepatocytes that are adjacent to the connective tissue of the portal triads [13]. Serial sections have revealed that the nerve fibers arise from nerve bundles in the intrahepatic bile ducts [13]. Electron microscopy has shown that arterioles and bile ducts are in direct contact with these nerve fibers [11]. Liver innervation comprises sympathetic, parasympathetic, and peptidergic nerve fibers, organized as either afferent or efferent nerves [14]. In human liver, nerve endings in the intralobular spaces are located mainly in the Disse spaces and are oriented toward the hepatic stellate cells, sinusoidal endothelial cells, and hepatocytes [15]. This study evaluated the topographical changes in the hepatic nerve by investigating S100B protein expression using immunohistochemistry, and semi-quantitatively by monitoring S100B mRNA 
expression gauged as the $S_{100 B / G A P D H}$ mRNA ratio, in chronic liver disease subjects, as compared to subjects with normal livers.

Quantification of the hepatic nerve in liver diseases has been reported in a few studies, but the results are still controversial. The density of the hepatic nerve has been measured in previous studies by calculating the number of positively stained cells in a microscopic field $[11,16,17]$. Nam et al. [11] reported a decreased density of the hepatic nerve in patients with chronic liver diseases, and this density was significantly lower in patients with LC than in normal controls. Terada and Nakanuma [15] reported a higher density of the hepatic nerve in hepatolithiasis and extrahepatic biliary obstruction than in normal human livers. Matsunaga et al. [18] reported that S1oo-positive nerve fibers in the stroma were significantly denser in patients with chronic liver disease than in those with normal livers. The stained nerve fibers were manually counted in the above-mentioned studies, so that errors in quantifying positively stained fibers might have affected the results.

In contrast, Terada and Nakanuma [15] reported that nerve fibers were dense in patients with hepatolithiasis, intermediate in patients with extrahepatic biliary obstruction, and sparse in normal controls. S1oo-positive nerve fibers in the stroma were significantly increased in patients with chronic liver diseases compared to those with normal livers, and the S1oo-positive nerve fiber density was significantly higher in patients with all types of chronic liver disease than in those with normal livers in yet another study [18], and was thought to be due to nerve regeneration during chronic liver inflammation $[16,18]$. The proliferated Schwann cells seemed to form a scaffold guiding regenerating nerve fibers [19]. Considering that the noradrenergic fibers run along blood vessels, changes in vascular patterns may also alter hepatic innervation patterns $[19,20]$. Moreover, scar formation, with changes in the microcirculatory dynamics could influence nerve regeneration [20], and changes in vascularity and inflammation can affect nerve expression $[19,21]$.

In our study, LC patients showed decreased hepatic nerve density in regenerating nodules compared to that of control group (Table 3), in accordance with previous studies $[2,11,22]$. Nam et al. [11] suggested that decreased hepatic innervation in LC and chronic hepatitis patients might be caused by progressive fibrosis. Nerve fibers were also completely absent in patients with advanced cirrhosis, suggesting that parenchymal nerves may be damaged by progressive fibrosis [22]. The presence of regenerating nodules, as an abnormal growth mode, may contribute to sinusoidal denervation and structural changes may be responsible for the lack of parenchymal nerves in cirrhosis and the relative abundance of nerves in fibrous septa [22].

As hepatic fibrosis occurs in the portal spaces and extends into the parenchyma, proliferated capillaries, together with fibrous tissues, fibrosis may invade the parenchymal remnants from the enlarged portal spaces, which may result in structural changes in the lobules in chronic liver disease [20] and which suggests that hepatic parenchymal nerve damage becomes more prominent as fibrosis progresses.

In our study, S100B mRNA expression was significantly lower in chronic liver disease than in the control group. However, within the chronic liver diseases group, the increase in the fibrosis score failed to show significant correlation with $S_{100 B}$ mRNA expression. From these findings, we cautiously suggest that a decrease of S10oB expression as a marker of hepatic nerve might be an early event of chronic liver diseases and fibrosis progression, as an early chronic hepatitis stage prior to progression to cirrhosis. Once the chronic liver diseases process is activated, the $\mathrm{S} 100 \mathrm{~B}$ expression seems not to change markedly, as the expression was not different among chronic liver diseases and was not correlated with fibrosis scores.

Structural changes in the lobules in patients with chronic liver diseases may also result in altered nerve fiber distribution [2]. Hepatic nerve density in regenerating nodules was significantly decreased in patients with LC in this study, and Miyazawa et al. [2] have previously reported that nerve fibers were decreased or altogether absent in the pseudolobules of cirrhotic liver, whereas they were increased markedly in fibrous septae. Jaskiewicz et al. [23] also reported that hepatic parenchymal nerves were absent in cirrhotic nodules and were depleted in the fibrotic strands of alcoholic cirrhotic livers. Using electron microscopy, we evaluated hepatic nerves in one normal control, and in nine $\mathrm{CHB}$, five $\mathrm{CHC}$, one LC, and two HCC patients and noted that the basement membrane of hepatic nerve ending was dis- 
rupted in patients with chronic hepatitis (Supplementary Fig. 1). Another study reported that unmyelinated nerve fiber bundles in the intralobular connective tissue coursed in the vicinity of hepatic triads [11]. Unmyelinated nerve fibers, including axons, mitochondria, and lysosomes were found in the vicinity of portal tract and bile duct cells in our specimens.

However, we were not able to correlate these findings with hepatic nerve density or S10oB expression. Further electron microscopic studies are warranted to clarify the meaning of these ultrastructural changes in chronic liver diseases.

There were several limitations to this study. The number of enrolled patients, and particularly the number of control samples was small, and all of them were women. The mean age of the patients was different between groups. The stained nerve fibers were manually counted, which could affect the results. However, our findings of decreased hepatic innervation in chronic liver diseases may facilitate understanding of chronic liver disease or liver fibrosis.

In conclusion, the S10oB/GAPDH mRNA ratio, and S1ooB protein expression, as a marker of hepatic nerves, was decreased in patients with chronic liver diseases and compared to the normal controls; hepatic nerve density was decreased in regenerating nodules in patients with LC. Decrease in S1ooB expression as a marker of hepatic nerves might be an early event in chronic liver disease progression, representing an early chronic hepatitis stage before progression to cirrhosis. Further studies are needed to clarify the association between hepatic innervation and structural changes in hepatic lobules.

\section{KEY MESSAGE}

1. The $S_{100 B / G A P D H}$ mRNA ratio, and $\mathrm{S}_{100 \mathrm{~B}}$ protein expression, as a marker of the hepatic nerve, is decreased in patients with chronic liver diseases.

2. Hepatic innervation is decreased in chronic liver disease patients, especially in the lobular portion, compared to the control group and seems to decrease in the early stage of fibrosis progression.

\section{Conflict of interest}

No potential conflict of interest relevant to this article was reported.

\section{REFERENCES}

1. Tiniakos DG, Lee JA, Burt AD. Innervation of the liver: morphology and function. Liver 1996;16:151-160.

2. Miyazawa Y, Fukuda Y, Imoto M, Koyama Y, Nagura H. Immunohistochemical studies on the distribution of nerve fibers in chronic liver diseases. Am J Gastroenterol 1988;83:1108-1114.

3. Henriksen JH, Moller S, Ring-Larsen H, Christensen NJ. The sympathetic nervous system in liver disease. J Hepatol 1998;29:328-341.

4. Hartmann H, Beckh K, Jungermann K. Direct control of glycogen metabolism in the perfused rat liver by the sympathetic innervation. Eur J Biochem 1982;123:521-526.

5. Gardemann A, Puschel GP, Jungermann K. Nervous control of liver metabolism and hemodynamics. Eur J Biochem 1992;207:399-411.

6. Donato R. Functional roles of S1oo proteins, calcium-binding proteins of the EF-hand type. Biochim Biophys Acta 1999;1450:191-231.

7. Ribeiro L, Andreazza AC, Salvador M, et al. Oxidative stress and $\mathrm{S}_{100 B}$ protein in cirrhotic children. Neurochem Res 2007;32:1600-1603.

8. Strauss GI, Christiansen M, Moller K, Clemmesen JO, Larsen FS, Knudsen GM. S-1oob and neuron-specific enolase in patients with fulminant hepatic failure. Liver Transpl 2001;7:964-970.

9. Donato R, Michetti F. Specific binding sites for S-100 protein in isolated brain nuclei. J Neurochem 1981;36:16981705.

10. Matus A, Mughal S. Immunohistochemical localisation of S-100 protein in brain. Nature 1975;258:746-748.

11. Nam SW, Song HJ, Back SJ, et al. Decreased hepatic nerve fiber innervation in patients with liver cirrhosis. Gut Liver 2007;1:165-170.

12. Knodell RG, Ishak KG, Black WC, et al. Formulation and application of a numerical scoring system for assessing histological activity in asymptomatic chronic active hepatitis. Hepatology 1981;1:431-435.

13. Yamada E. Some observations on the nerve terminal on the liver parenchymal cell of the mouse as revealed 
by electron microscopy. Okajimas Folia Anat Jpn 1965;40:663-677.

14. Streba LA, Vere CC, Ionescu AG, Streba CT, Rogoveanu I. Role of intrahepatic innervation in regulating the activity of liver cells. World J Hepatol 2014;6:137-143.

15. Terada T, Nakanuma Y. Innervation of intrahepatic bile ducts and peribiliary glands in normal human livers, extrahepatic biliary obstruction and hepatolithiasis: an immunohistochemical study. J Hepatol 1989;9:141-148.

16. Ueno T, Bioulac-Sage P, Balabaud C, Rosenbaum J. Innervation of the sinusoidal wall: regulation of the sinusoidal diameter. Anat Rec A Discov Mol Cell Evol Biol 2004;280:868-873.

17. Terada T, Matsunaga Y. S-100-positive nerve fibers in hepatocellular carcinoma and intrahepatic cholangiocarcinoma: an immunohistochemical study. Pathol Int 2001;51:89-93.

18. Matsunaga Y, Kawasaki H, Terada T. Stromal mast cells and nerve fibers in various chronic liver diseases: rele- vance to hepatic fibrosis. Am J Gastroenterol 1999;94:19231932.

19. Ungvary G, Donath T. Changes of the peripheral autonomic nervous system in altered internal environment. Z Mikrosk Anat Forsch 1980;94:985-998.

20. Rappaport AM, MacPhee PJ, Fisher MM, Phillips MJ. The scarring of the liver acini (Cirrhosis): tridimensional and microcirculatory considerations. Virchows Arch A Pathol Anat Histopathol 1983;402:107-137.

21. Fukuda Y, Nagura H, Imoto M, Koyama Y. Immunohistochemical studies on structural changes of the hepatic lobules in chronic liver diseases. Am J Gastroenterol 1986;81:1149-1155.

22. Lee JA, Ahmed Q, Hines JE, Burt AD. Disappearance of hepatic parenchymal nerves in human liver cirrhosis. Gut 1992;33:87-91.

23. Jaskiewicz K, Voigt MD, Robson SC. Distribution of hepatic nerve fibers in liver diseases. Digestion 1994;55:247252. 


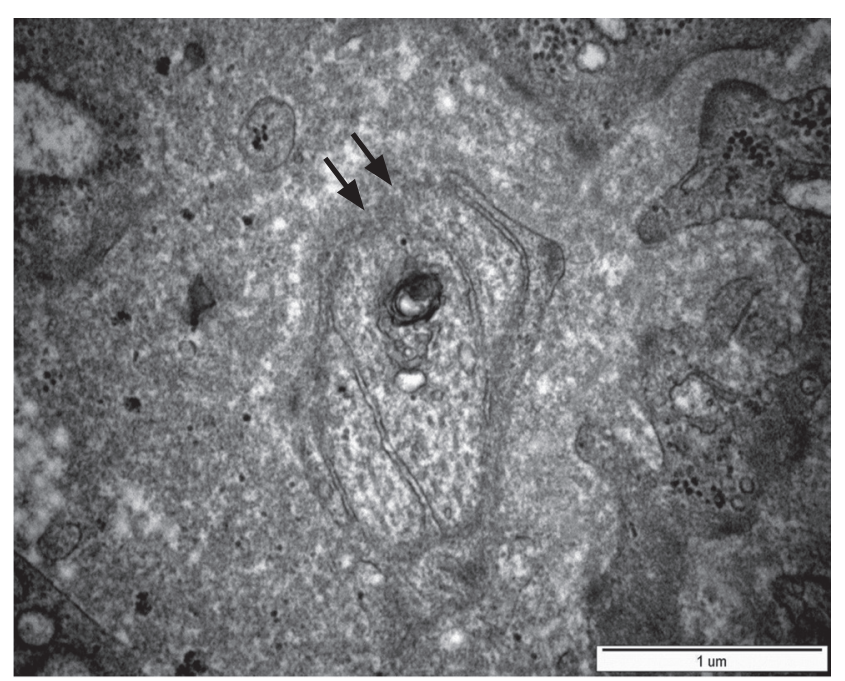

Supplementary Figure 1. Electron microscopic findings of the hepatic nerve. Disruption of basement membrane in hepatic nerve ending is indicated as arrows (chronic hepatitis B patient, $\times 50,000$ ). 